East African Medical Journal Vol. 77 No. 1 January 2000

OBSTRUCTED ABDOMINAL HERNIA AT THE WESLEY GUILD HOSPITAL, NIGERIA

A.R.K. Adesunkanmi, FMCS, FWACS, E.A. Agbakwuru, FMCS and T. A. Badmus, FWACS, Department of Surgery, College of Health Sciences, Obafemi Awolowo University, Ile-Ife, Nigeria.

Request for reprints to: Dr. A.R.K. Adesunkanmi, Department of Surgery, College of Health Sciences, Obafemi Awolowo University, Ile-Ife, Nigeria.

\title{
OBSTRUCTED ABDOMINAL HERNIA AT THE WESLEY GUILD HOSPITAL, NIGERIA
}

\author{
A.R.K. ADESUNKANMI, E.A. AGBAKWURU and T. A. BADMUS
}

\begin{abstract}
Objective: To determine the incidence, pattern and outcome of obstructed abdominal wall hernia in a semi-urban and rural community.

Design: A proforma was drafted to study all consecutive patients operated for obstructed anterior abdominal wall hernia over a period of five years. Clinical findings, preoperative treatment, operative findings and postoperative outcome were documented.

Setting: Teaching hospital located in a semi-urban community comprising mostly agrarian population.

Patients: A total 110 adult patients with obstructed anterior abdominal wall hernia who had operative intervention and other postoperative management.

Results: There were 110 patients with 111 obstructed hernias, accounting for $26.4 \%$ of all abdominal wall hernias. The age ranged from 19 - 79 years with mean of 49.7 years. Males accounted for $\mathbf{8 1 \%}$. Inguinoscrotal hernia was the commonest occurring in $75.7 \%, 16.2 \%$ patients presented with inguinal hernia and five patients with femoral hernia. The greater proportion of inguinal hernia occurred in female. Eighty seven patients $(79 \%)$ had emergency operations and elective in 23 patients $(21 \%)$ who had spontaneous reduction while awaiting surgery. Ninety two percent of inguinoscrotal/ inguinal hernia were indirect. Omentum was trapped in 52 hernias $(47 . \%)$, while in 15 patients $(13.6 \%)$, gangrenous bowel segments were discovered. Scrotal oedema was the commonest complication accounting for $21 \%$, while wound infection occurred in $20 \%$. There were three deaths in elderly men with clinical symptoms and signs of acute intestinal obstruction and gangrenous bowel segments, accounting for $2.7 \%$ of the patients. Twenty eight per cent of patients were discharged within the first and second postoperative days. Two patients spent 36 and 56 days each in the hospital.

Conclusion: This study showed that $26.4 \%$ of abdominal hernia presented with obstruction. With inguinoscrotal hernia predominating: male accounted for $81 \%$ and $13.6 \%$ of the obstructed hernia contained gangrenous bowel segments. Post-operative complications were common, mortality occurring mainly in elderly patients with late presentation.
\end{abstract}

\section{INTRODUCTION}

Obstruction is the commonest complication of abdominal wall hernias(1). Inguinal/inguinoscrotal hernias are the commonest. It accounts for between 50 and $78 \%$ of causes of acute intestinal obstruction in the tropics(2-4) and about $25 \%$ in the western world(5). Recent studies have, however, shown that prominence of groin hernias in acute intestinal obstruction may be diminishing(7). Obstructed groin hernias may be associated with poor prognosis, especially when they are associated with bowel strangulation and gangrene in elderly patients.

This study was carried out at the Wesley Guild Hospital, Ilesa, a component of Obafemi Awolowo University Teaching Hospitals Complex, (OAUTHC), Ile-Ife, Nigeria. It serves the largely agrarian population of rural and semi-urban communities of the Ijesas and Ekitis in western Nigeria. The aim was to determine the pattern and outcome of obstructed abdominal wall hernia in this environment and to elucidate the factors affecting prognosis.

\section{MATERIALS AND METHODS}

A proforma was drafted to study consecutive patients with obstructed abdominal wall hernias between $1991-1996$. All the cases with obstruction were included. All the patients were seen and assessed in the accident and emergency unit. Packed cell volumes were estimated in all the patients. Patients with features of acute intestinal obstruction had electrolytes and urea assessed. Initial resuscitation included intravenous fluids, nasogastric suction and administration of antibiotics in patients with features of ischaemic intestinal obstruction. All the patients were operated upon either under general or local anaesthesia with diazepam and/or pethidine. The operative methods used were either shouldice and 
modified Bassini, and MCVay procedures in groin hernias, Mayo double breasting procedure in ventral hernias.

Operative findings were noted, and postoperative course and findings were noted. The patients were followed up in the outpatient clinic after discharge.

Data were entered into Computer and analyzed using statistical package Epi-Info version 6 software.

\section{RESULTS}

There were 110 patients with 111 abdominal hernias that were diagnosed as obstructed at presentation. They presented at the accident and emergency unit. They accounted for $26.4 \%$ of all abdominal wall hernias seen over a period of five years. The ages ranged from 19-79 years with mean of 49.7 years \pm 12.5 . There were 90 males, accounting for $83 \%$ and 20 females (18\%), with the ratio of $4.5: 1$. Inguinoscrotal and inguinal hernias accounted for 102 patients (92.2\%), five patients had femoral hernias (4.5\%) and three cases had umbilical hernias. Fifty seven groin hernias were on the right side and 49 on the left, accounting for $51.8 \%$ and $44.5 \%$ respectively.

Inguinoscrotal hernia was the commonest, occurring in 85 patients, followed by inguinal hernia in 18 patients, accounting for $76 \%$ and $16.2 \%$ respectively. Most of the inguinal hernias occurred in females.

Irreducible groin and umbilical swellings were the commonest presentation, followed by abdominal pain and vomiting (Table 1). The duration of symptoms other than those of obstruction ranged from three months to 25 years with a mean of $2.73 \pm 4.47$ years. Obstructive symptoms occurred about one to five days before presentation.

\section{Table 1}

Clinical presentations and diagnoses in the 110 patients with 111 obstructed hernias

\begin{tabular}{lrr}
\hline Clinical presentation & No. of patients & $\%$ \\
\hline Irreducible swelling & 110 & 100 \\
Abdominal pain & 97 & 88 \\
Vomiting & 85 & 77.3 \\
Abdominal distension & 18 & 16.4 \\
Oth. assoc. clin. find & 19 & 17.3 \\
Diagnoses & - & - \\
Inguinoscrotal hernia & 85 & 76.6 \\
Inguinal hernia & 18 & 16.2 \\
Femoral hernia & 5 & 4.5 \\
Umbilical hernia & 3 & 2.7 \\
\hline
\end{tabular}

Emergency herniorrhaphy was carried out in 87 patients $(79 \%), 23$ patients who had spontaneous reduction while awaiting surgery had elective herniorrhaphy on the following elective list. The type of anaesthesia, and status of the operating surgeons were as documented in Table 2.
Table 2

The types of operation, anaesthesia and status of operating surgeon

\begin{tabular}{lcc}
\hline Operation & No. of patients & $\%$ \\
\hline Emergency & 87 & 79 \\
Elective & 23 & 21 \\
Anaesthesia: & & \\
General & 73 & 66.4 \\
Local anaesthesia & 36 & 33 \\
Spinal & 1 & 0.9 \\
Status of surgeons: & & \\
Consultant & 36 & 33 \\
Senior registrar & 57 & 52 \\
Registrar/House officer & 14 & 15.4 \\
\hline
\end{tabular}

Table 3

Operative findings and postoperative complications

\begin{tabular}{lrlr}
\hline Operative finding & \multicolumn{3}{l}{ Postoperative complications } \\
\hline Indirect hernia sac & 101 & Scrotal oedema & $23(21 \%)$ \\
Direct hernia sac & 6 & Wound infection/sinuses & $22(30 \%)$ \\
Femoral & 5 & Scrotal haematoma & $15(13.6 \%)$ \\
Pantaloons hernia sac & 2 & Hypertrophic scar & $3(2.7 \%)$ \\
Inguinal hernia sac & & & \\
$\quad$ with interstial & 2 & Wound dehiscence & $2(1.8 \%)$ \\
Small intestine viable & 18 & Wound mal-apposition & $1(0.9 \%)$ \\
Small intestine gangrenous & 15 & Scrotal infection & $1(0.9 \%)$ \\
Caecum/Terminal ileum & 10 & Scrotal gangrene & $1(0.9 \%)$ \\
Omentum & 9 & Damaged vas & $1(0.9 \%)$ \\
Bladder & 5 & Forgotten foreign body & $3(2.7 \%)$ \\
Scrotal abscess & 1 & Death & $3(2.7 \%)$ \\
Hydrocele of the cord & 1 & & \\
\hline
\end{tabular}

Operative findings were mainly indirect hernia sacs in $96(86.5 \%)$ and direct sacs in only six patients. Table 3 shows postoperative findings and complications. The duration of hospital stay ranged from one to 56 days with a mean of $5.6 \pm 3.3$. Twenty six patients were discharged the same day, 15 patients spent two days, 46 patient were admitted for three to seven days, 16 patients spent eight to 14 days, five patients spent 15 to 21 days while one patient each spent 36 and 56 days.

\section{DISCUSSION}

Obstruction can lead to strangulation, a serious complication of hernias. The incidence ranges from 9 to $12 \%$ in inguinal hernias and $28 \%$ in femoral hernias(8). In this study it accounted for $28.4 \%$, with a $22.5 \%$ incidence of intestinal ischaemia and gangrene confirmed at surgery.

Generally in groin hernias more males were affected than females in a ratio of about 8:1, however, a larger proportion of females presented with obstruction. The male to female ratio in this study was 4.5:1 comparable to the ratio of 5:1 and 6:1 reported in the previous studies. The proportion of females is larger still when femoral hernias are considered, it may be as high as 3:1 and even a reversed ratio of about 1:7 in favour of females have been reported(9,10). Most studies(1,3,8-10) have reported the preponderance of obstruction and strangulation in elderly patients, similar to the findings in this study. 
The diagnosis of obstructed hernia is not difficult, although detection of intestinal ischaemia may be difficult preoperatively. Two rare strangulations, namely, Ritcher's hernia and Litres's hernia are more difficult to diagnose. In Ritcher's hernia a part of the intestinal circumference is involved in herniation with little or no symptoms of intestinal strangulation and poor local signs. In Litre's hernia, the Meckel's diverticulum is involved. Pain resulting from intra-abdominal diseases such as acute appendicitis, cholecystitis, generalised peritonitis and other causes of acute abdomen and symptomatic hernia must be distinguished from strangulating groin hernias.

In the tropics many patients present with features of acute intestinal obstruction secondary to inguinal hernia. Some will not volunteer the history of irreducible groin swelling until asked or discovered during clinical examination. Few patients may deny that they have been aware of the presence of groin swelling $(2-4,7,10)$. The distribution of obstructed hernias, the type and sides are in keeping with general distribution of hernias. Although inguinoscrotal hernia is the commonest in obstructed hernia, it requires immediate surgical attention as delay may lead to intestinal ischaemia.

Surgery is the treatment of choice, although gentle manipulative reduction has been advocated to reduce the incidence of intestinal ischaemia that may occur as a result of delay in surgical intervention. This approach has its side effects as ischaemic or gangrenous intestine may be reduced into the abdomen, also reduction en-masse may occur $(8,10)$. Apart from these, in our environment successful reduction may lead patients to seek discharge and may never be seen until another episode of obstruction occurs. Spontaneous reduction is however, possible after analgesic and sedation. Application of cold pack can also hasten spontaneous reduction.

The choice of anaesthesia depends on the clinical status of the patients, but local anaesthesia is adequate and most suitable for elderly patients with high risk of operation under general anaesthesia $(11,12)$.

Simple approach to the hernia repair has been recommended with direct incision to open the inguinal canal and prudent opening of the sac to prevent reduction of sac content into the peritoneal cavity so that it can be examined and its degree of intestinal ischaemia determined. Herniolaparotomy and laparotomy as suggested by some authors(8) in cases of sac content retraction is said to be unnecessary, the medial aspect of the neck of the sac can be widened to gain access and retrieve the retracted hernial sac content(10). Herniolaparotomy and laparotomy was not indicated in any of the patients in this study. The retracted hernia sac content, whenever such occurred, was easily retrieved with finger or tissue forceps through the internal ring. As in the previous studies, small intestine, large intestine and omentum were common abdominal contents in decreasing order of frequency.

The morbidity and mortality of obstructed hernia is related to the age of the patients and delayed presentation(5,12,13). Fatal outcome is significantly associated with old age, the need for bowel resection which is closely correlated with the time interval between the onset of acute symptoms and subsequent operation $(5,6,8,12,13)$. For strangulated hernia the mortality can be as high as $10-17 \%$, with the need for intestinal resection, it may be as high as $21-40 \%$ and as high and $33 \%$ in strangulated femoral hernia(8). Ajao(10) reported $2.6 \%$ mortality similar to the finding in this study. Other causes of morbidity also tend to occur in higher proportion in elderly patients.

In conclusion, obstructed hernia is very common in our environment. It is still responsible for a significant proportion of acute intestinal obstruction. It is commonest in indirect inguinoscrotal hernia, occasional in direct inguinoscrotal and femoral hernia. It occurred more in male patients, although a higher proportion of females was affected when compared to reducible abdominal hernias. The mortality is significant, mostly in elderly patients requiring intestinal resection. Early presentation and elective repair of abdominal wall hernia will eliminate the morbidity and mortality associated with this very common problems.

\section{ACKNOWLEDGEMENTS}

We are grateful to the residents who operated on some of these patients. We acknowledge the contribution of those who assisted during the preparation of this manuscript. We thank Dr. Abdul Rashid Aderinoye, Adult Education Department, University of Ibadan, for allowing access to his personal computer.

\section{REFERENCES}

1. Gallegos, N.C., Dawson, J., Jarvis, M. and Hobsley, M. Risk of strangulation in groin hernia. Brit. J. Surg. 1991; 78:11711173 .

2. Otu, A.A. Tropical surgical abdominal emergencies: Acute intestinal obstruction. Postgrad. Doct. 1992; 14:51-54.

3. Odigie, V.I., Muhammed, I. and daRocha-Afodu, J.T. Mechanical intestinal strangulating obstruction: The Zaria experience of 104 consecutive patients. Nig. J. Surg. 1996; 3:1-6.

4. Datubo-Brown, D.D., Adoter, J.M. Pattern of surgical acute abdomen. West Afr. J. Med. 1990; 9:59-62.

5. McEntes, G., Pender, D. and Mulum, D. et al. Current spectrum of intestinal obstruction. Brit. J. Surg. 1987; 74:59-62.

6. Andrew, N.J. Presentation and outcome of strangulated external hernia in a district general hospital. Brit. J. Surg. 1981; 68: 329-32.

7. Adesunkanmi, A.R.K. and Agbakwuru, E.A. The changing pattern of acute intestinal obstruction in Tropical African population. East Afr. Med. J. 1996; 11:726-730.

8. Stoppa, R.E. The treatment of complicated groin and incisional hernia. Wld. J. Surg. 1989; 13:545-554

9. Askew, G., Williams, G.T. and Brown, S.C. Delay in presentation and miss-diagnosis of strangulated hernia. A prospective study. J. Roy. coll. Surg. Edinb. 1992; 37:37-39.

10. Ajao, O.G. Obstructed groin hernia in a tropical African population. J. Natn. Med. Assoc. 1979; 71:1093-1094.

11. Behnia, R., Hashemi, F., Stryker, J.S., Ujiki, G.T. and Poticha, S.M. A comparison of general versus local anaesthesia during inguinal herniorrhaphy. Surg. gynecol. Obstet. 1992; 174:277-280.

12. Allen, P.I.M., Zager, M. and Goldman, M. Elective repair of groin hernias in the elderly. Brit. J. Surg. 1987; 74:987-90.

13. Levin, D.C., Moran, C.G. and Vellacott, K.D. Inguinal hernia repair in elderly patients. J. roy. coll. Surg. Edinb. 1989; 34:101-3. 